\title{
Osteomyelitis in Children, What to do?
}

\author{
Rasmus Hesthave Pedersen and Rikke Thorninger* \\ Randers Regional Hospital, Randers, Denmark \\ ${ }^{\star}$ Corresponding author: Rikke Thorninger, Assistant Professor, Randers Regional Hospital, Randers, Denmark; Email: rikkthor@rm.dk
}

Received: January 11, 2019; Accepted: January 19, 2019; Published: January 22, 2019;

\section{Introduction}

Osteomyelitis is defined as inflammation of the bone with subsequent bone destruction [1]. Osteomyelitis in children has been a diagnostic challenge for decades. Hematogenous osteomyelitis presents clinically versatile, depending on age and causative organism. Historically osteomyelitis has been classified by pathogenesis and duration. Osteomyelitis in children is primary of haematogenous origin [2-5] and was thought to originate from the metaphysis of long bones due to low blood flow in end capillaries [6]. Metaphyseal vessels are open ended towards the physis (growth plate) in long bones. Therefore Stephen RF. et al. [7] suggested the junctions between epiphysis and metaphysis to be the origin of infection in acute osteomyelitis. Time between onset of symptoms and confirmed diagnosis defines acute (within 14 days), subacute (within 3 months) and chronic osteomyelitis (more than 3 months) $[8,9]$.

Osteomyelitis in children most often originates from long bones, the lower extremities being the most common site, distributed as femur (23-29\%), tibia (19-26\%) and fibula (4-10\%) [5, 9].

Fractures and bony malignancies as Ewing's sarcoma are important differential diagnoses to rule out during initial assesment. Complications of untreated osteomyelitis in children are septic arthritis, growth retardation due to damage to the physis, bone deformation, angular deformity, septicemia, organ failure and death $[5,10,11]$.

\section{Diagnostics}

The diagnostic process is multimodal. Both biochemistry and image modalities are required to support the diagnosis sufficiently. Especially imaging modalities has evolved over recent years enabling detailed information on infection status. [3]

A systematic review on acute heamatogenous osteomyelitis [8] showed elevated C-reactive protein (CRP) in $80.5 \%$ of children on admission on presentation, $91 \%$ had raised Erythrocyte Sedimentation Rate (ESR) and 35.9\% had leukocytosis. Pääkönen M. et al evaluated inflammatory markers in 265 children with septic arthritis, osteomyelitis or both. They found sensitivity of ESR and CRP to be $94 \%$ and $95 \%$ respectively. Raised CRP and ESR simultaneously have a sensitivity of $98 \%$ for osteoarticular infection [12]. CRP has short half-life and is inexpensive which makes it useful for monitoring treatment $[8,9]$.

Subacute osteomyelitis presents with mild symptoms and often no positive laboratory findings [13]. Several studies [13, 14] have presented case series of subacute osteomyelitis in children, with low sensitivities of CRP, ESR and leukocytes.

Standard radiographs have differential diagnostic value, whereas early diagnosis of infection is dependent on more dynamic image modalities. Visible infection on plain radiographs is seen two to three weeks after onset of symptoms [9].

Magnetic resonance imaging (MRI) is the preferred modality for detecting primary focus of infection and it can be helpful in planning surgery. Several studies have documented sensitivity of $82-100 \%$ and specificity of $75-99 \%[10,15]$

Ultra Sonography can reveal subperiosteal changes, evaluate soft tissue and joint effusion and hence have a role in supporting the diagnosis [5].

Bone scans can be useful in multifocal disease and when no clear origin of infection has been found. In neonates specificity is lower due to higher rate of false negative scans $[5,8]$. In a recent study, white blood cell scintigraphy was found convincing in detecting post traumatic osteomyelitis [16] Another study reported sensitivity of 79\% and a specificity of $97 \%$ in detecting fracture related bony infection in peripheral bones [17].

\section{Microbiology and Antibiotics}

Staphylococcus Aureus, streptococcus and gram-negative organisms are primary causative organisms in young children [8]. In recent years the facultative anaerobic Gram-negative bacillus Kingella Kingae has been recognized as among the most common pathogen in children between six months and four years of age with haematogenous osteomyelitis $[4,6]$. Several published case series includes patients, in which no bacteriological causative organism is identified by microbiological tests on blood, pus or bone biopsy [11, $18]$.

Antibiotic treatment regimes of acute osteomyelitis in children have historically been $4-6$ weeks in total $[3,5]$. Two systematic reviews find short intravenous course (3-4 days) followed by 3 weeks of oral therapy as effective as longer intravenous treatment regimes, in uncomplicated cases of acute osteomyelitis. $[8,19]$ A recent open review concludes that definite guidelines for treatment length and route of administration are yet to be established [4].

Initial empirical treatment, if prevalence of Methicillin Sensitive Staphylococcus Aureus (MSSA) $>90 \%$, is in several studies recommended as short course intravenous antistaphylococcal 
penicillin. Benzylpenicillin/cephalosporin is added if patient is not immunized against Haemophilus Influenzae. When clinical improvement and lowered inflammatory markers treatment is finished by oral regime 3-4 weeks [3-5, 8]. In Methicillin Resistant Staphylococcus Aureus (MRSA) endemic areas, prevalence $>10 \%$ in community Clindamycin or Vancomycin are recommended $[3,9]$.

\section{Case Presentation}

A nine year old boy was referred to our outpatient clinic from the general practitioner, with plain radiographic findings suspicious of bony malignancy in left proximal fibula (Figure 1). The patient was otherwise healthy and had followed standard Danish children vaccine program.
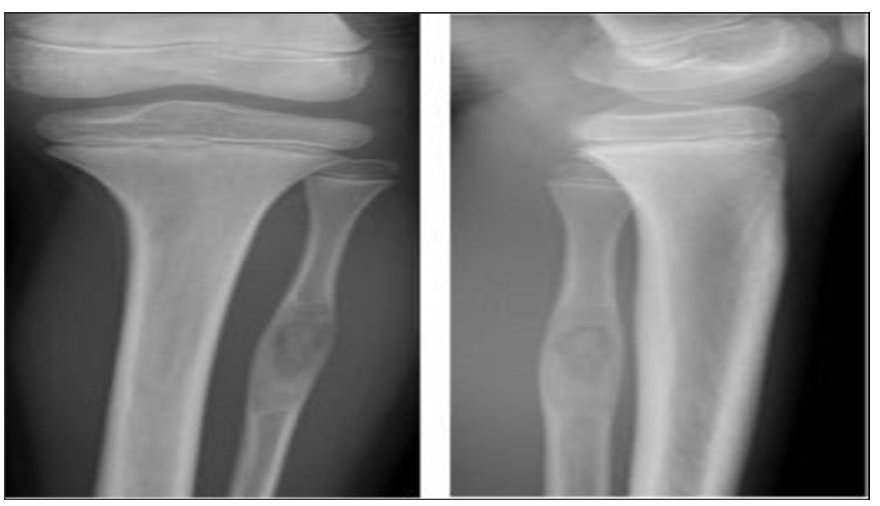

Figure 1. Primary plain x-ray when patient was admitted to our department showing isolated process in left proximal fibula.

The boy had 4 weeks lasting constant pain in proximal fibula of the left leg. There had been a minor contusion to the left knee prior to onset of pain. There had been no fewer, swelling or redness of the left leg at any time during period of pain.

Physical examination revealed slim inconspicuously looking legs with no difference between sides. Inspection showed no difference between healthy and affected leg. Palpation of the proximal fibula was painful.

Left leg movement was free and painless, and the neurovascular status was normal. X-ray and subsequently magnetic resonance scanning and computed tomography scan showed large sequester in the left proximal fibula (Figure 3), arising suspicion of sub-acute osteomyelitis. Blood sample inflammatory makers was all within normal range and there were no systemic signs of infection.

The sequester in left fibula was surgically removed with additional decortication above affected bone and clearing of the bone marrow canal. A Gentamicin implant was placed in the bone defect. The pathologic bone was microscopically evaluated and cultured. Cultures were all negative, microscopy showed no signs of acute or chronic inflammation. Morphology was found suspicious of osteofibrous dysplasia. Subsequent multidisciplinary team conference, at Aarhus University Hospital concluded that radiographic material and history suggested subacute osteomyelitis. Osteofibrous dysplasia and malignancy were excluded.

The patient underwent 14 days intravenous treatment postoperatively, with benzylpenicillin and dicloxacillin. After discharge treatment was completed with 4 weeks of per oral dicloxacillin. Amoxycillin/ Clauvulanic acid was primary per oral choice, but was stopped due to allergic skin reaction presenting within five days. Throughout the treatment period all blood tests were within normal range.

At three month follow up, the patient had recovered and had complete remission of pain. Radiographs showed healing in the defect area (Figure 2).
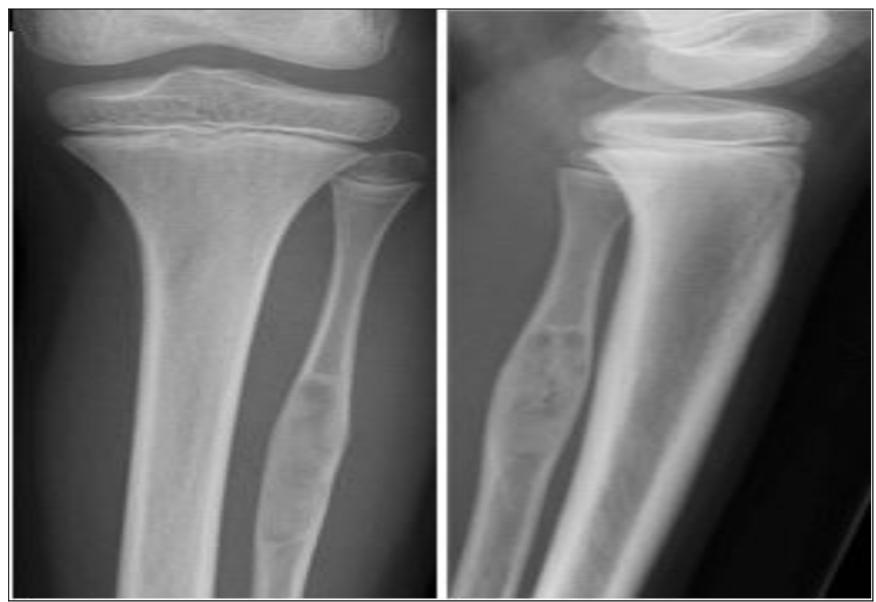

Figure 2. Plain radiographs three months after surgery showed healing around bony defect in proximal fibula.

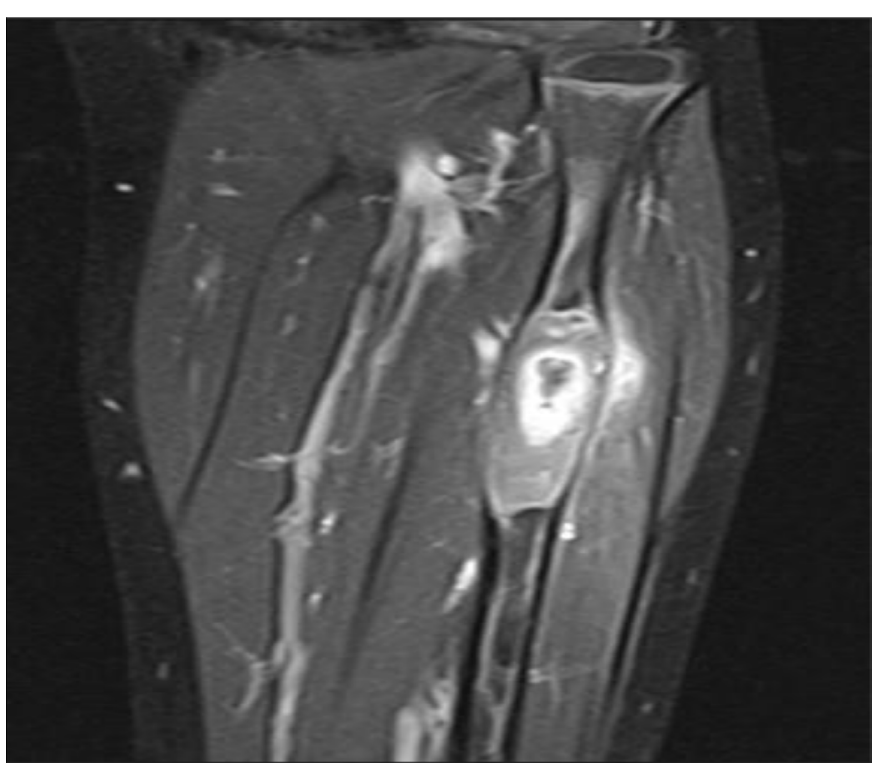

Figure 3. Magnetic resonance image prior to sugery, showing sequestra in left proximal fibula.

\section{Discussion}

In the presented case, a multidisciplinary team discussed paraclinical findings and sustained subacute osteomyelitis as tentative diagnosis, although blood samples had been normal and there were no sign of inflammation in sequestral bone biopsies. In a retrospective study with 121 children diagnosed with acute haematogenous osteomyelitis, sensitivity of blood culture was $32.4 \%$ and sensitivity of biopsy culture was $46.6 \%$. In this study $16.5 \%$ of patients included 
underwent surgery [11] Several studies suggest that surgery is reserved for those not responding to standard treatment, but may provide early microbiological diagnose and accelerate patient recovery [3-5]

The patient of this case was offered surgery and subsequently antibiotic therapy. The clinical follow up showed full recovery and remission of symptoms. Inflammatory markers, CRP, leukocytes and ESR was unchanged and within normal range. As pointed out in several studies $[3,4,8,20]$ there are no definite guidelines or clear consensus for assessment and treatment strategy for acute or especially subacute osteomyelitis in children. In this case treatment for suspected osteomyelitis was effectuated without final radiographic or microbiological diagnosis. Total remission of symptoms was seen within the first week after surgery, and there were no clinical sign of infection local or systemic.

The results of this treatment strategy were early recovery and minimal delay due to further diagnostic processes.

Bone abscess representing subacute osteomyelitis can clinically and radiographically mimic bony malignant tumors, being and important differential diagnosis [21]. Dhanoa A. et al. [20] presented six cases, including one child and three adolescents, with initial suspicion of bony malignancy. All cases where diagnosed with subacute osteomyelitis, confirmed by histopathological exam of needle biopsies and microbiological test. The diagnostic process represents a potential delay in relevant treatment of subacute osteomyelitis, and has been described as a clinical and diagnostic challenge $[13,20]$.

Our patient underwent surgical removal and in total 6 weeks of antibiotic treatment, although no microbiological diagnosis was obtained and biopsy showed no clear sign of osteomyelitis. For subacute osteomyelitis we recommend short antibiotic therapy, initial intravenously and shift to oral therapy guided primarily on clinical remission rather than laboratory testing. In cases with radiographically well defined abscesses, surgically debridement and cleansing of affected bone might support faster recovery and shorter antibiotic treatment regimes.

Further studies are needed to define and test diagnostic algorithms to support clinical decision making and minimize diagnostic delay.

\section{References}

1. de Graaf H, Sukhtankar P, Arch B, et al (2017) Duration of intravenous antibiotic therapy for children with acute osteomyelitis or septic arthritis: a feasibility study. Health Technol Assess 21: 1-164. [crossref]

2. Schmitt SK (2017) Osteomyelitis. Infect Dis Clin North Am 31: 325-338. [crossref]

3. Harik NS, MS Smeltzer (2010) Management of acute hematogenous osteomyelitis in children. Expert Rev Anti Infect Ther 8: 175- 181.

4. Iliadis AD, Ramachandran M (2017) Paediatric bone and joint infection. EFORT Open Rev 2: 7-12. [crossref]

5. Yeo A, Ramachandran M (2014) Acute haematogenous osteomyelitis in children. BMJ 348: 66. [crossref]

6. Jaramillo D, et al (2017) Hematogenous Osteomyelitis in Infants and Children: Imaging of a Changing Disease. Radiology 283: 629-643.

7. Stephen RF, MK Benson, S Nade (2012) Misconceptions about childhood acute osteomyelitis. J Child Orthop 6: 353-356.

8. Dartnell J, M Ramachandran, M Katchburian (2012) Haematogenous acute and subacute paediatric osteomyelitis: a systematic review of the literature. J Bone Joint Surg Br 94: 584-595.

9. Peltola H, Pääkkönen M (2014) Acute osteomyelitis in children. N Engl J Med 370: 352-360. [crossref]
10. van Schuppen J, MM van Doorn, RR van Rijn (2012) Childhood osteomyelitis: imaging characteristics. Insights Imaging 3: 519-533.

11. Chiappini E, et al (2017) Epidemiology and Management of Acute Haematogenous Osteomyelitis in a Tertiary Paediatric Center. Int J Environ Res Public Health 14(5).

12. Paakkonen M, et al (2010) Sensitivity of erythrocyte sedimentation rate and Creactive protein in childhood bone and joint infections. Clin Orthop Relat Res 468: 861-866.

13. Spyropoulou V, et al (2016) Primary subacute hematogenous osteomyelitis in children: a clearer bacteriological etiology. J Child Orthop 10: 241-246.

14. Foster CE, et al (2018) Brodie's Abscess in Children: A Ten--Year Single Institution Retrospective Review. Pediatr Infect Dis J 2018

15. Thevenin--Lemoine C, et al (2016) MRI of acute osteomyelitis in long bones of children: Pathophysiology study. Orthop Traumatol Surg Res 102: 831-837.

16. Govaert GA, IJpma FF, McNally M, McNally E, et al (2017) Accuracy of diagnostic imaging modalities for peripheral post-traumatic osteomyelitis - a systematic review of the recent literature. Eur J Nucl Med Mol Imaging 44: 1393-1407. [crossref]

17. Govaert, GAM, et al (2018) High diagnostic accuracy of white blood cel scintigraphy for fracture related infections: Results of a large retrospective single-center study. Injury 49: 1085-1090.

18. Floyed, RL, RW Steele (2003) Culture negative osteomyelitis. Pediatr Infect Dis $J$ 22: 731-736.

19. Howard--Jones, AR, D Isaacs (2013) Systematic review of duration and choice of systemic antibiotic therapy for acute haematogenous bacterial osteomyelitis in children. J Paediatr Child Health 49: 760-768.

20. Dhanoa A, VA Singh (2010) Subacute osteomyelitis masquerading as primary bone sarcoma: report of six cases. Surg Infect (Larchmt) 11: 475-478.

21. McCarville MB (2009) The child with bone pain: malignancies and mimickers. Cancer Imaging 9: 115-121. [crossref]

\section{Citation:}

Pedersen RH, Thorninger R (2018) Osteomyelitis in Children, What to do?. Integr J Orthop Traumatol Volume 2(1): 1-3 